\title{
ON SOME LINEAR OPERATORS ON FOCK TYPE SPACE
}

\author{
I.KH. MUSIN
}

\begin{abstract}
We consider a lower semi-continuous function $\varphi$ in $\mathbb{R}^{n}$ depending on the absolute values of the variables and growing faster than $a \ln (1+\|x\|)$ for each positive $a$. In terms of this function, we define a Hilbert space $F_{\varphi}^{2}$ of entire functions in $\mathbb{C}^{n}$. This is a natural generalization of a classical Fock space. In this paper we provide an alternative description of the space $F_{\varphi}^{2}$ in terms of the coefficients in the power expansions for the entire functions in this space. We mention simplest properties of reproducing kernels in the space $F_{\varphi}^{2}$. We consider the orthogonal projector from the space $L_{\varphi}^{2}$ of measurable complex-valued functions $f$ in $\mathbb{C}^{n}$ such that

$$
\|f\|_{\varphi}^{2}=\int_{\mathbb{C}^{n}}|f(z)|^{2} e^{-2 \varphi(\operatorname{abs} z)} d \mu_{n}(z)<\infty
$$

where $z=\left(z_{1}, \ldots, z_{n}\right)$, abs $z=\left(\left|z_{1}\right|, \ldots,\left|z_{1}\right|\right)$, on its closed subspace $F_{\varphi}^{2}$, and for this projector we obtain an integral representation.

We also obtain an integral formula for the trace of a positive linear continuous operator on the space $F_{\varphi}^{2}$. By means of this formula we find the conditions, under which a weighted operator of the composition on $F_{\varphi}^{2}$ is a Hilbert-Schmidt operator. Two latter results generalize corresponding results by Sei-Ichiro Ueki, who studied similar questions for operators in Fock space.
\end{abstract}

Keywords: entire functions, Fock type space, linear operators, operator trace, weighted composition operators, Hilbert-Schmidt operator.

Mathematics Subject Classification: 32A15, 42B10, 46E22, 47B33

\section{INTRODUCTION}

1.1. On considered problems. Let $H\left(\mathbb{C}^{n}\right)$ be a space of entire functions in $\mathbb{C}^{n}$ equipped with a topology of uniform convergence on compact subset $\mathbb{C}^{n}, d \mu_{n}$ be the Lebesgue measure in $\mathbb{C}^{n}$, abs $u=\left(\left|u_{1}\right|, \ldots,\left|u_{n}\right|\right)$ for $u=\left(u_{1}, \ldots, u_{n}\right) \in \mathbb{R}^{n}\left(\mathbb{C}^{n}\right)$.

By $V\left(\mathbb{R}^{n}\right)$ we denote the set of lower-semi-continuous function $v: \mathbb{R}^{n} \rightarrow \mathbb{R}$ obeying the conditions:

$\left.i_{1}\right) . v(x)=v($ abs $x), x \in \mathbb{R}^{n}$;

$\left.i_{2}\right) . \lim _{x \rightarrow \infty} \frac{v(x)}{\ln (1+\|x\|)}=+\infty$;

$\left.i_{3}\right)$. The restriction of $v$ on $[0, \infty)^{n}$ does not decrease in each variable.

Given $\varphi \in V\left(\mathbb{R}^{n}\right)$, by $L_{\varphi}^{2}$ we denote the space of measurable functions $f: \mathbb{C}^{n} \rightarrow \mathbb{C}$ such that

$$
\|f\|_{\varphi}^{2}=\int_{\mathbb{C}^{n}}|f(z)|^{2} e^{-2 \varphi(a b s z)} d \mu_{n}(z)<\infty
$$

with the scalar product $(f, g)_{\varphi}=\int_{\mathbb{C}^{n}} f(z) \overline{g(z)} e^{-2 \varphi(a b s z)} d \mu_{n}(z), f, g \in L_{\varphi}^{2}$; the space $L_{\varphi}^{2}$ is Hilbert.

Let $F_{\varphi}^{2}=L_{\varphi}^{2} \cap H\left(\mathbb{C}^{n}\right)$. It is easy to show that $F_{\varphi}^{2}$ is a closed subspace of the space $L_{\varphi}^{2}$. By Condition $i_{2}$ ), the polynomials belong to $F_{\varphi}^{2}$.

The definition of the space $F_{\varphi}^{2}$ is a natural generalization of the Fock space and it motivates a study of a series of problems related both the theory of functions and the operator theory.

I.Kh. Musin, On SOME Linear operators on Fock type SPACE.

(c) I.KH. Musin. 2018.

Submitted August 24, 2018. 
In the present paper we provide an alternative description of the space $F_{\varphi}^{2}$ and we restrict ourselves by considering an operator of orthogonal projection from $L_{\varphi}^{2}$ into $F_{\varphi}^{2}$ and by finding the conditions under which a weighted composition operator from $F_{\varphi}^{2}$ into $F_{\varphi}^{2}$ is a Hilbert-Schmidt operator.

1.2. Notations. Given $u=\left(u_{1}, \ldots, u_{n}\right), v=\left(v_{1}, \ldots, v_{n}\right) \in \mathbb{R}^{n}\left(\mathbb{C}^{n}\right)$, we let $\langle u, v\rangle:=u_{1} v_{1}+\cdots+$ $u_{n} v_{n},\|u\|$ is the Euclidean norm in $u$.

For $\varphi \in V\left(\mathbb{R}^{n}\right), \alpha=\left(\alpha_{1}, \ldots, \alpha_{n}\right) \in \mathbb{Z}_{+}^{n}, z=\left(z_{1}, \ldots, z_{n}\right) \in \mathbb{C}^{n}|\alpha|:=\alpha_{1}+\ldots+\alpha_{n}$ is the length of a multi-index $\alpha, \tilde{\alpha}:=\left(\alpha_{1}+1, \ldots, \alpha_{n}+1\right), z^{\alpha}:=z_{1}^{\alpha_{1}} \cdots z_{n}^{\alpha_{n}}$,

$$
c_{\alpha}(\varphi):=\int_{\mathbb{C}^{n}}\left|z_{1}\right|^{2 \alpha_{1}} \cdots\left|z_{n}\right|^{2 \alpha_{n}} e^{-2 \varphi(a b s z)} d \mu_{n}(z), e_{\alpha}(z)=\frac{z^{\alpha}}{\sqrt{c_{\alpha}(\varphi)}} .
$$

Given $R>0$, by $\Pi_{R}$ we denote the polydisk $\left\{z \in \mathbb{C}^{n}:\left|z_{1}\right|<R, \ldots,\left|z_{n}\right|<R\right\}$.

For a function $u$ with the domain containing the set $(0, \infty)^{n}$, we define a function $u[e]$ in $\mathbb{R}^{n}$ by the rule: $u[e](x)=u\left(e^{x_{1}}, \ldots, e^{x_{n}}\right), x=\left(x_{1}, \ldots, x_{n}\right) \in \mathbb{R}^{n}$.

The Young-Fenchel transform of the function $u: \mathbb{R}^{n} \rightarrow[-\infty,+\infty]$ is a function $u^{*}: \mathbb{R}^{n} \rightarrow$ $[-\infty,+\infty]$ defined by the formula $u^{*}(x)=\sup _{y \in \mathbb{R}^{n}}(\langle x, y\rangle-u(y)), x \in \mathbb{R}^{n}$.

Given a Hilber space $H$, by $(f, g)_{H}$ we denote the scalar product in $H$, while $\|x\|_{H}$ stands for the Hilbert norm of an element $x \in H$. Instead of $(f, g)_{F_{\varphi}^{2}}$ we write $(f, g)_{\varphi}$.

1.3. Main results. Theorem 1 provides a description of entire functions forming the space $F_{\varphi}^{2}$ in terms of the coefficients in their power expansions. The simplest properties of the reproducing kernels of the space $F_{\varphi}^{2}$ are given in Section 2. An explicit form for the orthogonal projector from $L_{\varphi}^{2}$ into $F_{\varphi}^{2}$ is obtained in Theorem 3. In Section 3 we obtain an integral formula for the trace of a linear continuous operator on $F_{\varphi}^{2}$, see Theorem 4. It is employed in proof of Theorem 5 , in which there formulated the conditions, under which a weighted composition operator on $F_{\varphi}^{2}$ is a Hilbert-Schmidt operator. Theorem 5 generalizes the main result of work [2], in which weighted compositions operators were considered in the Fock space. Its proof follows the main line of that of Theorem 1 in [2].

\section{SpaCe $F_{\varphi}^{2}$}

\subsection{Preliminaries.}

Proposition. Let $\varphi \in V\left(\mathbb{R}^{n}\right)$. Then

$$
\begin{aligned}
& (\varphi[e])^{*}(x)<+\infty \quad \text { for } \quad x \in[0, \infty)^{n}, \quad \lim _{\substack{x \rightarrow \infty \\
x \in[0, \infty)^{n}}} \frac{(\varphi[e])^{*}(x)}{\|x\|}=+\infty, \\
& c_{\alpha}(\varphi) \geqslant \frac{\pi^{n}}{\tilde{\alpha}_{1} \cdots \tilde{\alpha}_{n}} e^{2(\varphi[e])^{*}(\tilde{\alpha})}, \quad \alpha \in \mathbb{Z}_{+}^{n} .
\end{aligned}
$$

Proof. The first statement can be proved by straightforward calculations.

For each $x \in[0, \infty)^{n}$ and $t \in \mathbb{R}^{n}$ we have $(\varphi[e])^{*}(x) \geqslant\langle x, t\rangle-(\varphi[e])(t)$. In particular, this implies that for each $M>0$

$$
(\varphi[e])^{*}(x) \geqslant M\|x\|-\varphi[e]\left(\frac{M x}{\|x\|}\right) \geqslant M\|x\|-\varphi\left(e^{M}, \ldots, e^{M}\right), \quad x \in[0, \infty)^{n} \backslash\{0\} .
$$

This proves the second statement.

The third statement can be proved by an approach used in the proof of Lemma 2 in [3].

Corollary. Let $\varphi \in V\left(\mathbb{R}^{n}\right)$. Then for each $M>0$ there exists a constant $C_{M}>0$ such that $c_{\alpha}(\varphi) \geqslant C_{M} M^{|\alpha|}$ for each $\alpha \in \mathbb{Z}_{+}^{n}$.

\subsection{Orthonormal basis in $F_{\varphi}^{2}$.}

Theorem 1. Let $\varphi \in V\left(\mathbb{R}^{n}\right)$ and an entire function $f(z)=\sum_{|\alpha| \geqslant 0} a_{\alpha} z^{\alpha}$ belongs to $F_{\varphi}^{2}$. Then

$$
\sum_{|\alpha| \geqslant 0}\left|a_{\alpha}\right|^{2} c_{\alpha}(\varphi)<\infty, \quad\|f\|_{\varphi}^{2}=\sum_{|\alpha| \geqslant 0}\left|a_{\alpha}\right|^{2} c_{\alpha}(\varphi) .
$$


Vice versa, let a sequence $\left(a_{\alpha}\right)_{|\alpha| \geqslant 0}$ of complex numbers $a_{\alpha}$ is such that the series $\sum_{|\alpha| \geqslant 0}\left|a_{\alpha}\right|^{2} c_{\alpha}(\varphi)$ converges. Then $f(z)=\sum_{|\alpha| \geqslant 0} a_{\alpha} z^{\alpha} \in H\left(\mathbb{C}^{n}\right)$ and $f \in F_{\varphi}^{2}$.

Proof. Let $f(z)=\sum_{|\alpha| \geqslant 0} a_{\alpha} z^{\alpha} \in H\left(\mathbb{C}^{n}\right)$. The chain of identities

$$
\begin{aligned}
\|f\|_{\varphi}^{2} & \left.=\int_{\mathbb{C}^{n}}|f(z)|^{2} e^{-2 \varphi(a b s} z\right) d \mu_{n}(z)=\lim _{R \rightarrow \infty} \int_{\Pi_{R}}|f(z)|^{2} e^{-2 \varphi(a b s z)} d \mu_{n}(z) \\
& =\lim _{R \rightarrow \infty} \int_{\Pi_{R}} \sum_{|\alpha| \geqslant 0} a_{\alpha} z^{\alpha} \sum_{|\beta| \geqslant 0} \bar{a}_{\beta} \bar{z}^{\beta} e^{-2 \varphi(a b s z)} d \mu_{n}(z) \\
& =\lim _{R \rightarrow \infty} \sum_{\alpha, \beta \in \mathbb{Z}_{+}^{n}} a_{\alpha} \bar{a}_{\beta} \int_{\Pi_{R}} z^{\alpha} \bar{z}^{\beta} e^{-2 \varphi(a b s z)} d \mu_{n}(z) \\
& =\lim _{R \rightarrow \infty} \sum_{|\alpha| \geqslant 0} \int_{\Pi_{R}}\left|a_{\alpha}\right|^{2}\left|z_{1}\right|^{2 \alpha_{1}} \ldots\left|z_{n}\right|^{2 \alpha_{n}} e^{-2 \varphi(a b s z)} d \mu_{n}(z) \\
& =\lim _{R \rightarrow \infty} \int_{\Pi_{R}} \sum_{|\alpha| \geqslant 0}\left|a_{\alpha}\right|^{2}\left|z_{1}\right|^{2 \alpha_{1}} \ldots\left|z_{n}\right|^{2 \alpha_{n}} e^{-2 \varphi(a b s z)} d \mu_{n}(z) \\
& =\int_{\mathbb{C}^{n}} \sum_{|\alpha| \geqslant 0}\left|a_{\alpha}\right|^{2}\left|z_{1}\right|^{2 \alpha_{1}} \ldots\left|z_{n}\right|^{2 \alpha_{n}} e^{-2 \varphi(a b s z)} d \mu_{n}(z) \\
& =\sum_{|\alpha| \geqslant 0}\left|a_{\alpha}\right|^{2} \int_{\mathbb{C}^{n}}\left|z_{1}\right|^{2 \alpha_{1}} \cdots\left|z_{n}\right|^{2 \alpha_{n}} e^{-2 \varphi(a b s z)} d \mu_{n}(z)=\sum_{|\alpha| \geqslant 0}\left|a_{\alpha}\right|^{2} c_{\alpha}(\varphi)
\end{aligned}
$$

shows that $f \in F_{\varphi}^{2}$ if and only if $\sum_{|\alpha| \geqslant 0}\left|a_{\alpha}\right|^{2} c_{\alpha}(\varphi)<\infty$.

Vice versa, the convergence of the series $\sum_{|\alpha| \geqslant 0}\left|a_{\alpha}\right|^{2} c_{\alpha}(\varphi)$ and Corollary yield that for each $\varepsilon>0$ there exists a number $c_{\varepsilon}>0$ such that $\left|a_{\alpha}\right| \leqslant c_{\varepsilon} \varepsilon^{|\alpha|}$ for each $\alpha \in \mathbb{Z}_{+}^{n}$. This means that $f(z)=\sum_{|\alpha| \geqslant 0} a_{\alpha} z^{\alpha}$ is an entire function in $\mathbb{C}^{n}$. The above identities imply that $f \in F_{\varphi}^{2}$.

Lemma 1. Let $\varphi \in V\left(\mathbb{R}^{n}\right)$ and an entire function $f(z)=\sum_{|\alpha| \geqslant 0} a_{\alpha} z^{\alpha}$ belongs to $F_{\varphi}^{2}$. Then $\left(f, e_{\alpha}\right)_{\varphi}=$ $a_{\alpha} \sqrt{c_{\alpha}(\varphi)}$ for each $\alpha \in \mathbb{Z}_{+}^{n}$.

Proof. For each $\alpha \in \mathbb{Z}_{+}^{n}$ we have

$$
\begin{aligned}
\left(f, e_{\alpha}\right)_{\varphi} & =\lim _{R \rightarrow \infty} \int_{\Pi_{R}} f(z) \overline{e_{\alpha}(z)} e^{-2 \varphi(a b s z)} d \mu_{n}(z) \\
& =\lim _{R \rightarrow \infty} \sum_{|\beta| \geqslant 0} a_{\beta} \sqrt{c_{\beta}(\varphi)} \int_{\Pi_{R}} e_{\beta}(z) \overline{e_{\alpha}(z)} e^{-2 \varphi(a b s z)} d \mu_{n}(z) \\
& =a_{\alpha} \sqrt{c_{\alpha}(\varphi)} \lim _{R \rightarrow \infty} \int_{\Pi_{R}}\left|e_{\alpha}(z)\right|^{2} e^{-2 \varphi(a b s z)} d \mu_{n}(z) \\
& =a_{\alpha} \sqrt{c_{\alpha}(\varphi)} \int_{\mathbb{C}^{n}}\left|e_{\alpha}(z)\right|^{2} e^{-2 \varphi(a b s z)} d \mu_{n}(z)=a_{\alpha} \sqrt{c_{\alpha}(\varphi)} .
\end{aligned}
$$

Lemma 2. Let $\varphi \in V\left(\mathbb{R}^{n}\right)$. Then the system $\left\{e_{\alpha}\right\}_{\alpha \in \mathbb{Z}_{+}^{n}}$ is an orthonormal basis in $F_{\varphi}^{2}$.

Proof. The system $\left\{e_{\alpha}\right\}_{\alpha \in \mathbb{Z}_{+}^{n}}$ is orthogonal in $F_{\varphi}^{2}$. Indeed, for each $\alpha, \beta \in \mathbb{Z}_{+}^{n}: \alpha \neq \beta$ we have

$$
\int_{\Pi_{R}} e_{\alpha}(z) \overline{e_{\beta}(z)} e^{-2 \varphi(a b s z)} d \mu_{n}(z)=0, \quad R>0,
$$


and hence,

$$
\left(e_{\alpha}, e_{\beta}\right)_{\varphi}=\int_{\mathbb{C}^{n}} e_{\alpha}(z) \overline{e_{\beta}(z)} e^{-2 \varphi(a b s z)} d \mu_{n}(z)=\lim _{R \rightarrow \infty} \int_{\Pi_{R}} e_{\alpha}(z) \overline{e_{\beta}(z)} e^{-2 \varphi(a b s z)} d \mu_{n}(z)=0 .
$$

It is obvious that $\left\|e_{\alpha}\right\|_{\varphi}=1$ for each $\alpha \in \mathbb{Z}_{+}^{n}$.

An orthonormal in $F_{\varphi}^{2}$ system $\left\{e_{\alpha}\right\}_{\alpha \in \mathbb{Z}_{+}^{n}}$ is complete since by Theorem 1 and Lemma 1 for each

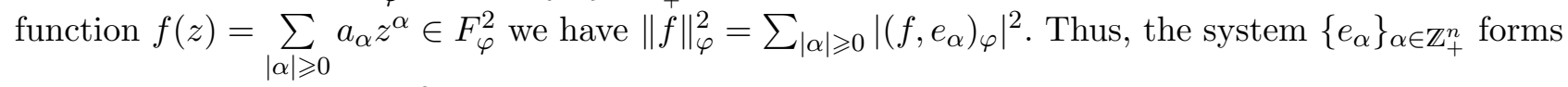
an orthonormal basis in $F_{\varphi}^{2}$.

2.3. Reproducing kernels for $F_{\varphi}^{2}$. We define a function $\mathcal{K}: \mathbb{C}^{2 n} \rightarrow \mathbb{C}$ by the rule

$$
\mathcal{K}(z, w)=\sum_{|\alpha| \geqslant 0} \frac{z^{\alpha} w^{\alpha}}{c_{\alpha}(\varphi)}, z, w \in \mathbb{C}^{n} .
$$

Since by Corollary for each $M>0$ there exists a constant $C_{M}>0$ such that

$$
c_{\alpha}(\varphi) \geqslant C_{M} M^{|\alpha|}, \quad \alpha \in \mathbb{Z}_{+}^{n},
$$

it is clear that $\mathcal{K} \in H\left(\mathbb{C}^{2 n}\right)$.

For $z \in \mathbb{C}^{n}$ we define the function $\mathcal{K}_{z}: \mathbb{C}^{n} \rightarrow \mathbb{C}$ by the rule $\mathcal{K}_{z}(w)=\mathcal{K}(z, w)$.

Lemma 3. Let $\varphi \in V\left(\mathbb{R}^{n}\right), z \in \mathbb{C}^{n}$. Then $\mathcal{K}_{z} \in F_{\varphi}^{2}$, and $\left\|\mathcal{K}_{z}\right\|_{\varphi}^{2}=\mathcal{K}(z, \bar{z})$.

Proof. By $\nu$ we denote the measure in $\mathbb{C}^{n}$ defined by the rule $d \nu(z)=e^{-2 \varphi(a b s z)} d \mu_{n}(z)$. Let $z \in \mathbb{C}^{n}$. For each $R>0$ we have

$$
\begin{aligned}
\int_{\Pi_{R}}\left|\mathcal{K}_{z}(w)\right|^{2} d \nu(w) & =\int_{\Pi_{R}} \sum_{|\alpha| \geqslant 0} \frac{z^{\alpha} w^{\alpha}}{c_{\alpha}(\varphi)} \sum_{|\beta| \geqslant 0} \frac{\bar{z}^{\beta} \bar{w}^{\beta}}{c_{\beta}(\varphi)} d \nu(w) \\
& =\sum_{\alpha, \beta \in \mathbb{Z}_{+}^{n}} \frac{z^{\alpha}}{c_{\alpha}(\varphi)} \frac{\bar{z}^{\beta}}{c_{\beta}(\varphi)} \int_{\Pi_{R}} w^{\alpha} \bar{w}^{\beta} d \nu(w)=\sum_{|\alpha| \geqslant 0} \frac{\left|z^{\alpha}\right|^{2}}{c_{\alpha}^{2}(\varphi)} \int_{\Pi_{R}}\left|w^{\alpha}\right|^{2} d \nu(w) \\
& =\int_{\Pi_{R}} \sum_{|\alpha| \geqslant 0} \frac{\left|z^{\alpha}\right|^{2}\left|w^{\alpha}\right|^{2}}{c_{\alpha}^{2}(\varphi)} d \nu(w) .
\end{aligned}
$$

Therefore,

$$
\begin{aligned}
\left\|\mathcal{K}_{z}\right\|_{\varphi}^{2} & =\int_{\mathbb{C}^{n}}\left|\mathcal{K}_{z}(w)\right|^{2} d \nu(w)=\lim _{R \rightarrow \infty} \int_{\Pi_{R}} \sum_{|\alpha| \geqslant 0} \frac{\left|z^{\alpha}\right|^{2}\left|w^{\alpha}\right|^{2}}{c_{\alpha}^{2}(\varphi)} d \nu(w) \\
& =\int_{\mathbb{C}^{n}} \sum_{|\alpha| \geqslant 0} \frac{\left|z^{\alpha}\right|^{2}\left|w^{\alpha}\right|^{2}}{c_{\alpha}^{2}(\varphi)} d \nu(w)=\sum_{|\alpha| \geqslant 0} \frac{\left|z^{\alpha}\right|^{2}}{c_{\alpha}^{2}(\varphi)} \int_{\mathbb{C}^{n}}\left|w^{\alpha}\right|^{2} d \nu(w)=\sum_{|\alpha| \geqslant 0} \frac{\left|z^{\alpha}\right|^{2}}{c_{\alpha}(\varphi)} .
\end{aligned}
$$

Thus, $\mathcal{K}_{z} \in F_{\varphi}^{2}$ since by inequality (1) the series $\sum_{|\alpha| \geqslant 0} \frac{\left|z^{\alpha}\right|^{2}}{c_{\alpha}(\varphi)}$ converges uniformly on compact subsets in $\mathbb{C}^{n}$ and $\left\|\mathcal{K}_{z}\right\|_{\varphi}^{2}=\mathcal{K}(z, \bar{z})$.

Lemma 4. Let $\varphi \in V\left(\mathbb{R}^{n}\right)$. Then for all $\alpha \in \mathbb{Z}_{+}^{n}$ and $z \in \mathbb{C}^{n}$ the identities hold

$$
\left(e_{\alpha}, \mathcal{K}_{\bar{z}}\right)_{\varphi}=\int_{\mathbb{C}^{n}} e_{\alpha}(w) \mathcal{K}(z, \bar{w}) e^{-2 \varphi(a b s w)} d \mu_{n}(w)=e_{\alpha}(z)
$$

Proof. Let $\alpha \in \mathbb{Z}_{+}^{n}$ and $z \in \mathbb{C}^{n}$. Then for each $R>0$

$$
\begin{aligned}
\int_{\Pi_{R}} e_{\alpha}(w) \mathcal{K}(z, \bar{w}) e^{-2 \varphi(a b s w)} d \mu_{n}(w) & =\int_{\Pi_{R}} e_{\alpha}(w) \sum_{|\beta| \geqslant 0} e_{\beta}(z) \overline{e_{\beta}(w)} e^{-2 \varphi(a b s w)} d \mu_{n}(w) \\
& =\sum_{|\beta| \geqslant 0} e_{\beta}(z) \int_{\Pi_{R}} e_{\alpha}(w) \overline{e_{\beta}(w)} e^{-2 \varphi(a b s w)} d \mu_{n}(w)
\end{aligned}
$$


We obtain that

$$
=e_{\alpha}(z) \int_{\Pi_{R}}\left|e_{\alpha}(w)\right|^{2} e^{-2 \varphi(a b s w)} d \mu_{n}(w) .
$$

$$
\begin{aligned}
\int_{\mathbb{C}^{n}} e_{\alpha}(w) \mathcal{K}(z, \bar{w}) e^{-2 \varphi(a b s w)} d \mu_{n}(w) & =\lim _{R \rightarrow \infty} \int_{\Pi_{R}} e_{\alpha}(w) \mathcal{K}(z, \bar{w}) e^{-2 \varphi(a b s w)} d \mu_{n}(w) \\
& =e_{\alpha}(z) \lim _{R \rightarrow \infty} \int_{\Pi_{R}}\left|e_{\alpha}(w)\right|^{2} e^{-2 \varphi(a b s w)} d \mu_{n}(w) \\
& =e_{\alpha}(z) \int_{\mathbb{C}^{n}}\left|e_{\alpha}(w)\right|^{2} e^{-2 \varphi(a b s w)} d \mu_{n}(w)=e_{\alpha}(z) .
\end{aligned}
$$

Theorem 2. Let $\varphi \in V\left(\mathbb{R}^{n}\right)$. Then for each $f \in F_{\varphi}^{2}$

$$
f(z)=\int_{\mathbb{C}^{n}} f(w) \mathcal{K}(z, \bar{w}) e^{-2 \varphi(a b s w)} d \mu_{n}(w), z \in \mathbb{C}^{n} .
$$

Proof. Let $f \in F_{\varphi}^{2}$. For each $z \in \mathbb{C}^{n}$ we have

$$
\begin{aligned}
\int_{\mathbb{C}^{n}} f(w) \mathcal{K}(z, \bar{w}) e^{-2 \varphi(a b s w)} d \mu_{n}(w) & =\left(f, \mathcal{K}_{\bar{z}}\right)_{\varphi}=\left(\sum_{|\alpha| \geqslant 0}\left(f, e_{\alpha}\right)_{\varphi} e_{\alpha}, \mathcal{K}_{\bar{z}}\right)_{\varphi} \\
& =\sum_{|\alpha| \geqslant 0}\left(f, e_{\alpha}\right)_{\varphi}\left(e_{\alpha}, \mathcal{K}_{\bar{z}}\right)_{\varphi}=\sum_{|\alpha| \geqslant 0}\left(f, e_{\alpha}\right)_{\varphi} e_{\alpha}(z)=f(z) .
\end{aligned}
$$

Remark 1. For each $f \in F_{\varphi}^{2}$, thanks to the plurisubharmonicity of $|f|^{2}$ we have

$$
|f(z)|^{2} \leqslant \frac{1}{\nu_{n}(1)} \int_{\|w-z\| \leqslant 1}|f(w)|^{2} d \mu_{n}(w), \quad z \in \mathbb{C}^{n},
$$

where $\nu_{n}(1)$ is the volume of the unit ball in $\mathbb{C}^{n}$. Therefore,

$$
|f(z)|^{2} \leqslant \frac{1}{\nu_{n}(1)} \exp \left(\sup _{\|w-z\| \leqslant 1} 2 \varphi(\text { abs } w)\right)\|f\|_{\varphi}^{2} .
$$

By estimate (2), for each $z \in \mathbb{C}^{n}$, a linear functional $\delta_{z}: F_{\varphi}^{2} \rightarrow \mathbb{C}$ acting by the rule $\delta_{z}(f)=f(z)$ is continuous and therefore, there exists the unique function $K_{z} \in F_{\varphi}^{2}$ such that for each $f \in F_{\varphi}^{2}$ we have $f(z)=\left(f, K_{z}\right)_{\varphi}$. The functions $K_{z}\left(z \in \mathbb{C}^{n}\right)$ are called reproducing kernels for $F_{\varphi}^{2}$. At that, $K_{z}(w)=\mathcal{K}(\bar{z}, w)=\mathcal{K}_{\bar{z}}(w)$. In particular, this implies that $\left\|K_{z}\right\|_{\varphi}^{2}=\mathcal{K}(z, \bar{z})$.

\section{Special Classes of linear operators on $F_{\varphi}^{2}$}

\subsection{Orthogonal projector on $F_{\varphi}^{2}$.}

Theorem 3. Let $\varphi \in V\left(\mathbb{R}^{n}\right), P_{\varphi}: L_{\varphi}^{2} \rightarrow F_{\varphi}^{2}$ be an orthogonal projector. Then

$$
P_{\varphi}(f)(z)=\int_{\mathbb{C}^{n}} f(w) \mathcal{K}(z, \bar{w}) e^{-2 \varphi(a b s w)} d \mu_{n}(w), z \in \mathbb{C}^{n} .
$$

Proof. Let $f \in L_{\varphi}^{2}$, then $P_{\varphi}(f)$ can be represented as the series $P_{\varphi}(f)=\sum_{|\alpha| \geqslant 0}\left(f, e_{\alpha}\right)_{\varphi} e_{\alpha}$ converging in $F_{\varphi}^{2}$. For each $z \in \mathbb{C}^{n}$ we have

$$
\begin{aligned}
P_{\varphi}(f)(z) & =\sum_{|\alpha| \geqslant 0}\left(f, e_{\alpha}\right)_{\varphi} e_{\alpha}(z)=\left(f, \sum_{|\alpha| \geqslant 0} \overline{e_{\alpha}(z)} e_{\alpha}\right)_{\varphi} \\
& =\left(f, \mathcal{K}_{\bar{z}}\right)_{\varphi}=\int_{\mathbb{C}^{n}} f(w) \mathcal{K}(z, \bar{w}) e^{-2 \varphi(a b s w)} d \mu_{n}(w) .
\end{aligned}
$$


Remark 2. Identity (3) can be written as $P_{\varphi}(f)(z)=\left(f, K_{z}\right)_{\varphi}$.

\subsection{Trace of positive linear continuous operator on $F_{\varphi}^{2}$.}

Definition 1. A linear continuous operator $A$ on a Hilbert space $H$ is called positive if $(A x, x)_{H} \geqslant 0$ for each $x \in H$.

It is known [4, 12.32, Thm.] that a positive linear continuous operator $A$ on a Hilbert space $H$ is self-adjoin.

Definition 2. Let $H$ be a Hilbert space, $A$ be a positive linear continuous operator in $H$ and $\left\{\psi_{k}\right\}_{k \in \mathbb{N}}$ be an orthonormalized basis in $H$. The trace $\operatorname{tr}(A)$ of the operator $A$ is defined as $\operatorname{tr}(A)=$ $\sum_{k=1}^{\infty}\left(A\left(\psi_{k}\right), \psi_{k}\right)_{H}$

It is known [5, Lms. 5.6.2, 5.5.1] that the definition of the trace of an operator $A$ is independent on the basis in $H$.

Theorem 4. Let $A$ be a positive linear continuous operator on $F_{\varphi}^{2}$. Then

$$
\operatorname{tr}(A)=\int_{\mathbb{C}^{n}}\left(A\left(K_{z}\right), K_{z}\right)_{\varphi} e^{-2 \varphi(a b s z)} d \mu_{n}(z) .
$$

Proof. We have

$$
\begin{aligned}
& \int_{\mathbb{C}^{n}}\left(A\left(K_{z}\right), K_{z}\right)_{\varphi} e^{-2 \varphi(a b s z)} d \mu_{n}(z)=\lim _{R \rightarrow \infty} \int_{\Pi_{R}}\left(A\left(K_{z}\right), K_{z}\right)_{\varphi} e^{-2 \varphi(a b s z)} d \mu_{n}(z) \\
& =\lim _{R \rightarrow \infty} \int_{\Pi_{R}}\left(A\left(\sum_{|\alpha| \geqslant 0} \overline{e_{\alpha}(z)} e_{\alpha}\right), \sum_{|\beta| \geqslant 0} \overline{e_{\beta}(z)} e_{\beta}\right)_{\varphi} e^{-2 \varphi(a b s z)} d \mu_{n}(z) \\
& =\lim _{R \rightarrow \infty} \int_{\Pi_{R}} \sum_{\alpha, \beta \in \mathbb{Z}_{+}^{n}} \overline{e_{\alpha}(z)} e_{\beta}(z)\left(A\left(e_{\alpha}\right), e_{\beta}\right)_{\varphi} e^{-2 \varphi(a b s z)} d \mu_{n}(z) \\
& =\lim _{R \rightarrow \infty} \sum_{\alpha, \beta \in \mathbb{Z}_{+}^{n}} \int_{\Pi_{R}} \overline{e_{\alpha}(z)} e_{\beta}(z)\left(A\left(e_{\alpha}\right), e_{\beta}\right)_{\varphi} e^{-2 \varphi(a b s z)} d \mu_{n}(z) \\
& =\lim _{R \rightarrow \infty} \sum_{|\alpha| \geqslant 0} \int_{\Pi_{R}}\left|e_{\alpha}(z)\right|^{2}\left(A\left(e_{\alpha}\right), e_{\alpha}\right)_{\varphi} e^{-2 \varphi(a b s z)} d \mu_{n}(z) \\
& =\lim _{R \rightarrow \infty} \int_{\Pi_{R}} \sum_{|\alpha| \geqslant 0}\left|e_{\alpha}(z)\right|^{2}\left(A\left(e_{\alpha}\right), e_{\alpha}\right)_{\varphi} e^{-2 \varphi(\text { abs } z)} d \mu_{n}(z) \\
& =\int_{\mathbb{C}^{n}} \sum_{|\alpha| \geqslant 0}\left|e_{\alpha}(z)\right|^{2}\left(A\left(e_{\alpha}\right), e_{\alpha}\right)_{\varphi} e^{-2 \varphi(a b s z)} d \mu_{n}(z) \\
& =\sum_{|\alpha| \geqslant 0}\left(A\left(e_{\alpha}\right), e_{\alpha}\right)_{\varphi} \int_{\mathbb{C}^{n}}\left|e_{\alpha}(z)\right|^{2} e^{-2 \varphi(a b s z)} d \mu_{n}(z) \\
& =\sum_{|\alpha| \geqslant 0}\left(A\left(e_{\alpha}\right), e_{\alpha}\right)_{\varphi}=\operatorname{tr}(A) .
\end{aligned}
$$

Remark 3. We note that a trace formula of such kind for a positive linear continuous operator in the Bergman space on the unit circle was provided in work 1], see Proposition 6.3.2, while for the case of a Fock space of the functions of many variables it was given in work [2], see Lemma 1.

\subsection{Weighted composition operator on $F_{\varphi}^{2}$. .}

Definition 3. Let $H$ be a Hilbert space and $\left\{\psi_{k}\right\}_{k \in \mathbb{N}}$ be an orthonormalized basis in $H$. A linear continuous operator $A: H \rightarrow H$ is called a Hilbert-Schmidt operator if $\sum_{k=1}^{\infty}\left\|A\left(\psi_{k}\right)\right\|_{H}^{2}<\infty$. 
It is known [5, Lm. 5.5.1] that sum of the series is independent on the basis in $H$.

Theorem 5. Let a holomorphic mapping $h: \mathbb{C}^{n} \rightarrow \mathbb{C}^{n}$ and a function $u \in H\left(\mathbb{C}^{n}\right)$ are such that the linear operator $u C_{h}: f \in F_{\varphi}^{2} \rightarrow u(f \circ h)$ is continuous on $F_{\varphi}^{2}$. Then the following conditions are equivalent:

1) $u C_{h}$ is a Hilbert-Schmidt operator;

2) $\int_{\mathbb{C}^{n}}|u(z)|^{2} \mathcal{K}(h(z), \overline{h(z)}) e^{-2 \varphi(a b s z)} d \mu_{n}(z)<\infty$.

3) $\int_{\mathbb{C}^{n}}\left(\int_{\mathbb{C}^{n}}|u(w)|^{2}\left|K_{z}(h(w))\right|^{2} e^{-2(\varphi(a b s w)+\varphi(a b s z))} d \mu_{n}(w)\right) d \mu_{n}(z)<\infty$.

Proof. Conditions 1) and 2) are equivalent. Indeed, since

$$
\begin{aligned}
\sum_{|\alpha| \geqslant 0}\left\|u C_{h}\left(e_{\alpha}\right)\right\|_{\varphi}^{2} & =\sum_{|\alpha| \geqslant 0} \int_{\mathbb{C}^{n}}|u(z)|^{2} \frac{\left|h_{1}(z)\right|^{2 \alpha_{1}} \cdots\left|h_{n}(z)\right|^{2 \alpha_{n}}}{c_{\alpha}(\varphi)} e^{-2 \varphi(\text { abs } z)} d \mu_{n}(z) \\
& =\int_{\mathbb{C}^{n}}|u(z)|^{2} \sum_{|\alpha| \geqslant 0} \frac{\left|h_{1}(z)\right|^{2 \alpha_{1}} \cdots\left|h_{n}(z)\right|^{2 \alpha_{n}}}{c_{\alpha}(\varphi)} e^{-2 \varphi(a b s z)} d \mu_{n}(z) \\
& =\int_{\mathbb{C}^{n}}|u(z)|^{2} \mathcal{K}(h(z), \overline{h(z)}) e^{-2 \varphi(\text { abs } z)} d \mu_{n}(z),
\end{aligned}
$$

the operator $u C_{h}$ is Hilbert-Schmidt if and only if

$$
\int_{\mathbb{C}^{n}}|u(z)|^{2} \mathcal{K}(h(z), \overline{h(z)}) e^{-2 \varphi(a b s z)} d \mu_{n}(z)<\infty .
$$

Let us show that Conditions 1) and 3) are also equivalent. It is obvious that the operator $u C_{h}$ on $F_{\varphi}^{2}$ is Hilbert-Schmidt if and only if the trace of the operator $\left(u C_{h}\right)^{*} u C_{h}$ is finite. By Theorem 4 , this is true if and only if

And since

$$
\int_{\mathbb{C}^{n}}\left(\left(u C_{h}\right)^{*} u C_{h}\left(K_{z}\right), K_{z}\right)_{\varphi} e^{-2 \varphi(a b s z)} d \mu_{n}(z)<\infty .
$$

$$
\begin{aligned}
\left(\left(u C_{h}\right)^{*} u C_{h}\left(K_{z}\right), K_{z}\right)_{\varphi} & =\left(u C_{h}\left(K_{z}\right), u C_{h}\left(K_{z}\right)\right)_{\varphi} \\
& =\int_{\mathbb{C}^{n}}|u(w)|^{2}\left|K_{z}(h(w))\right|^{2} e^{-2 \varphi(a b s w)} d \mu_{n}(w),
\end{aligned}
$$

then $u C_{h}$ on $F_{\varphi}^{2}$ is a Hilbert-Schmidt operator if and only if

$$
\int_{\mathbb{C}^{n}}\left(\int_{\mathbb{C}^{n}}|u(w)|^{2}\left|K_{z}(h(w))\right|^{2} e^{-2(\varphi(a b s w)+\varphi(a b s z))} d \mu_{n}(w)\right) d \mu_{n}(z)<\infty .
$$

\section{BIBLIOGRAPHY}

1. K. Zhu. Operator theory in function spaces. Marcel Dekker, New York (1990).

2. Sei-Ichiro Ueki. Hilbert-Schmidt Weighted Composition Operator on the Fock space // Int. Journal of Math. Analysis, Vol. 1:16, 769-774 (2007).

3. I.Kh. Musin. On a Hilbert space of entire functions // Ufimskji Matem. Zhurn. 9:3, 111-118 (2017). [Ufa Math. J. 9:3, 109-117 (2017).]

4. W. Rudin. Functional analysis. McGraw-Hill Book Comp., New York (1973).

5. E. Brian Davies. Linear operators and their spectra. Cambridge Univ. Press, Cambridge (2007).

Ildar Khamitovich Musin,

Institute of Mathematics,

Ufa Federal Research Center, RAS,

Chernyshevskii str. 112,

450077, Ufa, Russia

E-mail: musin_ildar@mail.ru 